Supporting Information

\title{
Bipolar Exfoliation and In-situ Deposition of High-Quality Graphene for Supercapacitor Application
}

Iman Khakpour ${ }^{1}$, Amin Rabiei Baboukani ${ }^{1}$, Anis Allagui ${ }^{2}$, and Chunlei Wang ${ }^{1 *}$

${ }^{1}$ Department of Mechanical and Materials Engineering, Florida International University, Miami, FL33174, USA

${ }^{2}$ Department of Sustainable and Renewable Energy Engineering, University of Sharjah, Sharjah, UAE

To measure the thickness of the deposited rGO film on the negative electrode, a gold-coated glass was used as the feeding electrode. The processing time was increased to 72 hours to have more reliable measurement. The cross-sectional SEM image of the sample tilted by $45^{\circ}$ is shown in Figure S1. The thickness of resulted coating is about 700 to $800 \mathrm{~nm}$.

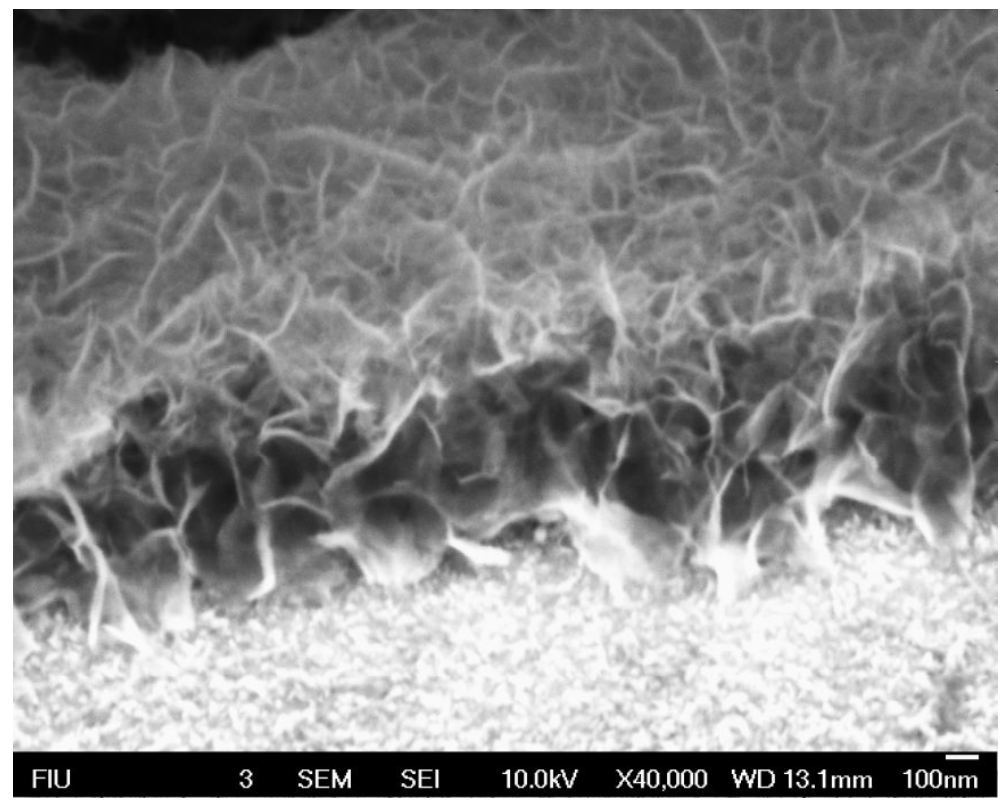

Figure S1. Cross-sectional SEM image (tilted by $45^{\circ}$ ) of the negative feeding electrode after 72 hours of BPE deposition. 


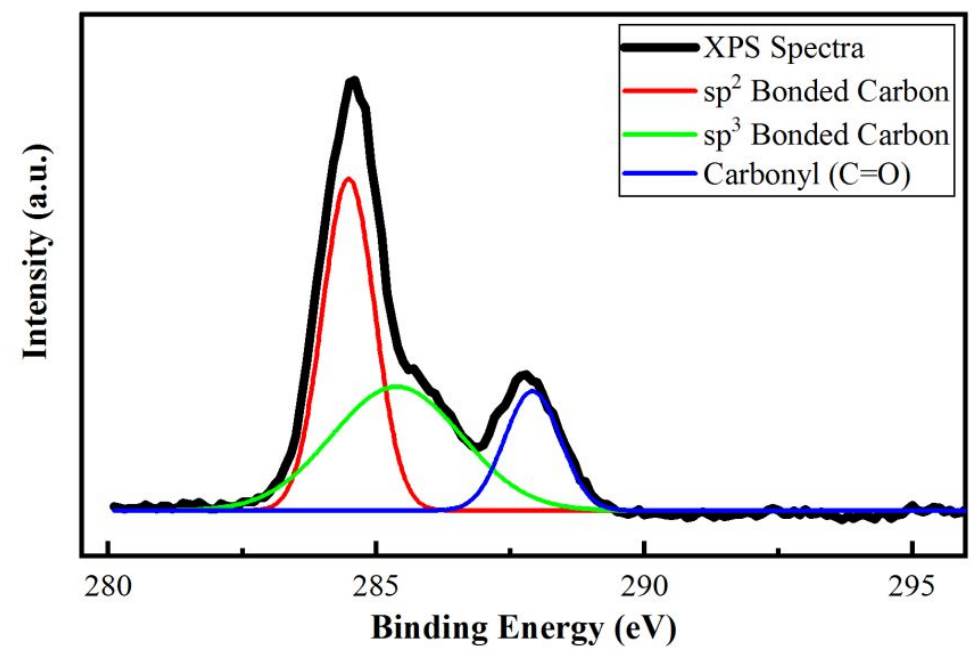

Figure S2. XPS (C1s) spectra of deposited material on negative feeding electrode. 